\title{
STABILITY OF THE FELLER PROPERTY FOR NON-LOCAL OPERATORS UNDER BOUNDED PERTURBATIONS
}

\author{
Yuichi Shiozawa and Toshiniro Uemura \\ Ritsumeikan University, Japan and University of Hyogo, Japan
}

ABSTRACT. It is known that the Feller property of a semigroup is stable under a bounded perturbation of the infinitesimal generator. Applying this, we derive the Feller property for a class of integro-differential operators including symmetric stable-like processes.

\section{INTRODUCTION}

Let $X$ be a locally compact separable metric space and $C_{\infty}(X)$ the Banach space of continuous functions on $X$ vanishing at infinity equipped with uniform norm. Let $A$ be the infinitesimal generator of a Feller semigroup on $C_{\infty}(X)$ and $B$ a bounded linear operator on $C_{\infty}(X)$. It is then known by [9, Theorem 3.7] that if $A+B$ is dissipative, then $A+B$ is again the infinitesimal generator of a Feller semigroup (see also Pazy ([17]) and Ethier and Kurtz ([10]) for related results). The purpose of this paper is to derive the Feller property for a class of non-local operators as an application of the stability result.

Since Fukushima ([11, Theorem 4.1]) established a one to one correspondence between regular Dirichlet forms and symmetric Hunt processes, the theory of Dirichlet forms has been one of the main tools to construct and study stochastic processes. However, as has already been pointed out in [19], the correspondence is only unique up to an equivalence. Here the equivalence is defined as follows: two symmetric Hunt processes corresponding to a regular Dirichlet form possess a common properly exceptional set outside of which

2010 Mathematics Subject Classification. 47G20, 60J75, 31C25.

Key words and phrases. Integro-differential operator, Feller semigroup, symmetric Dirichlet form of jump-type, stable-like process. 
their transition functions coincide ([12, Theorem 4.2.7]). This means that we have some ambiguity concerning starting points in constructing the process. So the question is left whether one can find a nice version from among an equivalence class of stochastic processes which start at each point in a natural way. Some conditions (e.g. Feller property or some kind of the strong Feller property) are known in order to construct stochastic processes from every starting point.

In 1988, Bass ([2]) succeeded in constructing a Feller process generated by the non-local operator $(-\Delta)^{\alpha(x) / 2}(0<\alpha(x)<2)$, the so-called stable-like process, by showing the existence and the uniqueness of the solution to the martingale problem. This process is a generalization of a symmetric $\alpha$-stable process. Motivated by this, the second author ([21]) (see also [22]) studied some path properties of the so-called symmetric stable-like process generated by the following regular Dirichlet form:

$$
\begin{aligned}
\mathcal{E}(u, v) & =\iint_{\mathbb{R}^{d} \times \mathbb{R}^{d} \backslash D} \frac{(u(x)-u(y))(v(x)-v(y))}{|x-y|^{d+\alpha(x)}} d x d y \\
\mathcal{F} & =\bar{C}_{0}^{\operatorname{lip}\left(\mathbb{R}^{d}\right)} \sqrt{\mathcal{E}_{1}(\cdot, \cdot)},
\end{aligned}
$$

where $C_{0}^{\text {lip }}\left(\mathbb{R}^{d}\right)$ stands for the set of all uniformly Lipschitz functions on $\mathbb{R}^{d}$ with compact support, $(u, v)_{L^{2}\left(\mathbb{R}^{d}\right)}=\int_{\mathbb{R}^{d}} u(x) v(x) d x$ and $\mathcal{E}_{1}(u, v)=\mathcal{E}(u, v)+$ $(u, v)_{L^{2}\left(\mathbb{R}^{d}\right)}$, and $D=\left\{(x, x) \in \mathbb{R}^{d} \times \mathbb{R}^{d}\right\}$. However, as symmetric stable-like processes are constructed by using Dirichlet form theory, we do not know whether they are Feller.

We are interested in when the semigroup corresponding to an infinitesimal generator or a (symmetric) Dirichlet form satisfies the Feller property. One possible way to show this property is to use potential theory. Bass and Levin ([6]) showed that harmonic functions associated with certain non-local operators are Hölder continuous by using the Harnack inequality and hitting time estimates. This implies the Hölder continuity of the resolvent of the operator. Since then, many authors have studied regularity of harmonic functions associated with non-local operators (see e.g. Chen and Kumagai ([8]), Song and Vondraček ([20]), Bass and Kassmann ([5]) and Schilling and Uemura $([19]))$. However, strong conditions are imposed on the jump kernels for the Harnack inequality to hold in those papers. Husseini and Kassmann [14] proved the Feller property of the resolvent corresponding to a jump-type symmetric Dirichlet form under a mild assumption for the kernel without using the Harnack inequality, but using some uniform continuity assumption for the kernel, what they called 'a priori estimate.' Though we do not use the estimate they assumed, our result is closely related to theirs.

Applying a dissipative perturbation, we will show the Feller property for the generators of certain jump-type symmetric Markov processes (Theorem 2.2 and Corollary 3.1). We will then obtain the Feller property for a 
class of symmetric stable-like processes, since the $L^{2}$-generator of a symmetric stable-like process is obtained from that of a stable-like process by a bounded perturbation. As a result, we can construct a symmetric stable-like process which is Feller (see Example 3.4).

We have originally learned the perturbation approach as mentioned above from $\S 4$ in [19]. There a given generator is recognized as the "small jumps" part perturbed by the "big jumps" part. They proved that the strong Feller property of the resolvent corresponding to the "small jumps" part implies the Feller property of the generator, while we assume the Feller property of a given operator.

It may seem curious that we derive the Feller property for the generator of a symmetric stable-like process through a (modified) non-symmetric stablelike process. In [23], we revealed a relation between $(-\Delta)^{\alpha(x) / 2}$ and the Dirichlet form $(\mathcal{E}, \mathcal{F})$ in (1.1) through the carré du champ operator associated with $(-\Delta)^{\alpha(x) / 2}$. There, we also found that the order of 'the principal (higher order) term' of the difference between the generator of the stable-like process and the $L^{2}$-generator of the Dirichlet form $(\mathcal{E}, \mathcal{F})$ is the same as that of the stable-like process (see [23, Theorem 3]). This is quite different from the case of diffusion processes. We can not reduce 'the principal term' to the perturbed operator by using a perturbation. Therefore, to show the Feller property for the perturbed operator, we would have to prove the Feller property and solve the martingale problem for it at the same time in general, and this means that it is not necessarily easier to study the symmetric non-local operator than to study the non-symmetric one.

\section{Perturbations by Bounded linear operators}

Let $X$ be a locally compact separable metric space and denote by $C_{\infty}(X)$ the Banach space of continuous functions on $X$ vanishing at infinity with norm $\|f\|_{\infty}=\sup _{x \in X}|f(x)|$. By a Feller semigroup we mean a strongly continuous, positive contraction semigroup on $C_{\infty}(X)$. Given a linear operator $A$ on $C_{\infty}(X)$, we denote its domain by $\mathcal{D}(A)$. We say that the operator $A$ is dissipative if

$$
\|\lambda u-A u\|_{\infty} \geq \lambda\|u\|_{\infty} \text { for all } u \in \mathcal{D}(A) \text { and all } \lambda>0 .
$$

As mentioned in $\S 1$, the following result is already known as a "dissipative perturbation" (see Theorem 3.7 in [9]):

Proposition 2.1. Let $A$ be the infinitesimal generator of a Feller semigroup on $C_{\infty}(X)$ and $B$ a bounded linear operator on $C_{\infty}(X)$. If $A+B$ is dissipative, then $A+B$ is again the infinitesimal generator of a Feller semigroup on $C_{\infty}(X)$. 
In what follows, we consider the following integro-differential operator:

$$
\mathcal{L} u(x)=\int_{y \neq x}(u(y)-u(x)) n(x, y) m(d y) \quad \text { for } u \in \mathcal{C} .
$$

More precisely, let $m$ be a positive Radon measure on $X$ with full support. Let $n$ be a positive measurable function defined on the off-diagonal set $\{(x, y) \in$ $X \times X: x \neq y\}$ such that $\mathcal{L} u \in C_{\infty}(X)$ for any $u \in \mathcal{C}$, where $\mathcal{C}$ is some dense subset in $C_{\infty}(X)$. Furthermore, we assume that $(\mathcal{L}, \mathcal{C})$ is closable on $C_{\infty}(X)$ and its closure is the infinitesimal generator of a Feller semigroup on $C_{\infty}(X)$. For instance, if $k(x, d y)=n(x, y) m(d y)$ is a bounded continuous kernel, then it is easy to see that $\mathcal{L}$ is a bounded linear operator on $C_{\infty}(X)$ satisfying the Feller property (see e.g. $[10, \S 8.3]$ ). Here we say that a kernel $k(x, d y)$ on $X$ is bounded if $k(x, d y)$ is a bounded measure on $X$ for any $x \in X$, and is continuous if $x \mapsto k(x, S)$ is a continuous function on $X$ for any Borel set $S \subset X$. We are also concerned with the following integro-differential operators:

$$
\mathcal{A} u(x)=\int_{y \neq x}(u(y)-u(x))(n(x, y)+n(y, x)) m(d y)
$$

and

$$
\tilde{\mathcal{L}} u(x)=\int_{y \neq x}(u(y)-u(x)) n(y, x) m(d y) .
$$

Here we note that even if $k(x, d y)=n(x, y) m(d y)$ is a bounded kernel, $\tilde{k}(x, d y):=n(y, x) m(d y)$ may be an unbounded kernel. Let $B$ be an integrodifferential operator defined by

$$
B u(x)=\int_{y \neq x}(u(y)-u(x))(n(y, x)-n(x, y)) m(d y) .
$$

We now state our main theorem:

TheOREm 2.2. Assume that $(\mathcal{L}, \mathcal{C})$ given by $(2.1)$ is closable on $C_{\infty}(X)$ and its closure is the infinitesimal generator of a Feller semigroup on $C_{\infty}(X)$. Assume further that the operator $B$ is a bounded linear operator on $C_{\infty}(X)$. Then $(\mathcal{A}, \mathcal{C})$ and $(\tilde{\mathcal{L}}, \mathcal{C})$ are also closable on $C_{\infty}(X)$ and their closures are the infinitesimal generators of Feller semigroups on $C_{\infty}(X)$, respectively.

Proof. Clearly $\mathcal{A}$ and $\tilde{\mathcal{L}}$ satisfy the positive maximum principle, and thus $\mathcal{A}$ and $\tilde{\mathcal{L}}$ are both dissipative. Now for $u \in \mathcal{C}$, we see that

$$
\begin{aligned}
\mathcal{A} u(x)= & \int_{y \neq x}(u(y)-u(x))(n(x, y)+n(y, x)) m(d y) \\
= & 2 \int_{y \neq x}(u(y)-u(x)) n(x, y) m(d y) \\
& +\int_{y \neq x}(u(y)-u(x))(n(y, x)-n(x, y)) m(d y)
\end{aligned}
$$


and

$$
\begin{aligned}
\tilde{\mathcal{L}} u(x)= & \int_{y \neq x}(u(y)-u(x)) n(y, x) m(d y) \\
= & \int_{y \neq x}(u(y)-u(x)) n(x, y) m(d y) \\
& +\int_{y \neq x}(u(y)-u(x))(n(y, x)-n(x, y)) m(d y) .
\end{aligned}
$$

We can then write down $\mathcal{A}$ and $\tilde{\mathcal{L}}$ as $\mathcal{A}=2 \mathcal{L}+B$ and $\tilde{\mathcal{L}}=\mathcal{L}+B$, respectively.

Since the closures of $\mathcal{L}$ and $2 \mathcal{L}$ on $C_{\infty}(X)$ are respectively the generators of Feller semigroups and $B$ is a bounded linear operator on $C_{\infty}(X)$ by assumption, we can extend both operators $\mathcal{A}$ and $\tilde{\mathcal{L}}$ to closed operators on $C_{\infty}(X)$ that are the infinitesimal generators of Feller semigroups by making use of Proposition 2.1.

Remark 2.3. Concerning the assumption on the operator $B$ in Theorem 2.2 , we impose a sufficient condition on $n$ in order that $B$ is indeed a bounded linear operator on $C_{\infty}(X)$ :

(1) for each $x$, the map $y \mapsto n(y, x)-n(x, y)$ is continuous on $X \backslash\{x\}$,

(2) there exists $\delta>1$ such that

$$
\sup _{x \in X}\left(\int_{y \neq x}|n(y, x)-n(x, y)| m(d y) \vee \int_{y \neq x}|n(y, x)-n(x, y)|^{\delta} m(d y)\right)<\infty,
$$

(3) there exists an increasing sequence of compact sets $\left\{F_{n}\right\}_{n=1}^{\infty}$ satisfying $m\left(X \backslash \bigcup_{n=1}^{\infty} F_{n}\right)=0$ such that for all compact sets $K \subset X$,

$$
\lim _{n \rightarrow \infty} \sup _{x \in K} \int_{F_{n}^{c}}|n(y, x)-n(x, y)| m(d y)=0,
$$

(4) for any $\varepsilon>0$ and compact set $K \subset X$, there exists a compact set $L \subset X$ with $K \subset L$ such that

$$
\sup _{x \in L^{c}} \sup _{x \in K}(n(x, y)+n(y, x))<\varepsilon .
$$

We can then show that, under Conditions (1)-(4), the operator $B$ is a bounded linear operator on $C_{\infty}(X)$. In the sequel, we fix $u \in C_{\infty}(X)$ so that $u \neq \equiv 0$ to avoid the triviality.

We first prove that $B u$ is a continuous function on $X$. To this end, fix $x_{\infty} \in X$ and take a sequence $\left\{x_{n}\right\}_{n=1}^{\infty} \subset X$ such that $x_{n} \rightarrow x_{\infty}$ as $n \rightarrow \infty$. Then there exists a compact set $K \subset X$ including $\left\{x_{n}\right\}_{n=1}^{\infty} \cup\left\{x_{\infty}\right\}$. Then it follows from Condition (3) that for any $\varepsilon>0$, there exists $N \in \mathbb{N}$ such that

$$
\sup _{x \in K} \int_{F_{N}^{c}}|n(y, x)-n(x, y)| m(d y)<\frac{\varepsilon}{4\|u\|_{\infty}} .
$$


For $n \in \mathbb{N} \cup\{\infty\}$ and $y \in X$, set

$$
\Phi_{n}(y):=\left(u(y)-u\left(x_{n}\right)\right)\left(n\left(y, x_{n}\right)-n\left(x_{n}, y\right)\right) .
$$

A direct calculation with (2.3) then implies that for any $n \in \mathbb{N}$,

$$
\begin{aligned}
& \left|B u\left(x_{n}\right)-B u\left(x_{\infty}\right)\right|=\left|\int_{y \neq x}\left(\Phi_{n}(y)-\Phi_{\infty}(y)\right) m(d y)\right| \\
& \leq \int_{F_{N}}\left|\Phi_{n}(y)-\Phi_{\infty}(y)\right| m(d y)+2\|u\|_{\infty} \int_{F_{N}^{c}}\left|n\left(y, x_{n}\right)-n\left(x_{n}, y\right)\right| m(d y) \\
& \quad+2\|u\|_{\infty} \int_{F_{N}^{c}}\left|n\left(y, x_{\infty}\right)-n\left(x_{\infty}, y\right)\right| m(d y) \\
& \quad \leq \int_{F_{N}}\left|\Phi_{n}(y)-\Phi_{\infty}(y)\right| m(d y)+\varepsilon .
\end{aligned}
$$

Moreover, we see from Condition (2) that

$$
\sup _{n \geq 1} \int_{F_{N}}\left|\Phi_{n}(y)\right|^{\delta} m(d y) \leq 2^{\delta}\|u\|_{\infty}^{\delta} \sup _{x \in X} \int_{F_{N}}|n(y, x)-n(x, y)|^{\delta} m(d y)<\infty
$$

that is, the sequence $\left\{\Phi_{n}\right\}_{n=1}^{\infty}$ is uniformly integrable with respect to the measure $m$ restricted on the compact set $F_{N}$. Since $\lim _{n \rightarrow \infty} \Phi_{n}(y)=\Phi_{\infty}(y)$ for any $y \neq x$ by the continuity of $u$ and Condition (1), the dominated convergence theorem leads us to

$$
\lim _{n \rightarrow \infty} \int_{F_{N}}\left|\Phi_{n}(y)-\Phi_{\infty}(y)\right| m(d y)=0,
$$

whence the continuity of $B u$ follows.

We finally show that $B u$ vanishes at infinity, that is, for any $\varepsilon>0$, there exists a compact set $K \subset X$ such that $|B u(x)|<\varepsilon$ for any $x \in K^{c}$. We see from Condition (2) that $M:=\sup _{x \in X} \int_{y \neq x}|n(y, x)-n(x, y)| m(d y)<\infty$. Since $u \in C_{\infty}(X)$ is a uniformly continuous function vanishing at infinity, for any $\varepsilon>0$, there exist a constant $\lambda>0$ and a compact set $K \subset X$ such that

$$
|u(x)-u(y)| \leq \frac{\varepsilon}{3 M} \quad \text { for any } x, y \in X \text { with } d(x, y) \leq \lambda
$$

and

$$
|u(x)|<\frac{\varepsilon}{3 M} \text { for any } x \in K^{c},
$$

where $d$ is a metric on $X$. In addition, Condition (4) yields that there exists a compact set $L$ with $K \subset L$ such that

$$
\sup _{x \in L^{c}} \sup _{y \in K}(n(x, y)+n(y, x))<\frac{\varepsilon}{3\|u\|_{\infty} m(K)} .
$$


Set $B_{x}(r)=\{y \in X \mid d(x, y) \leq r\}$ for $x \in X$ and $r>0$. Then for any $x \in L^{c}$,

$$
\begin{aligned}
|B u(x)| \leq & \int_{B_{x}(\lambda) \cap\{y \neq x\}}|u(y)-u(x)||n(y, x)-n(x, y)| m(d y) \\
& +\int_{B_{x}(\lambda)^{c}}|u(y)||n(y, x)-n(x, y)| m(d y) \\
& +|u(x)| \int_{B_{x}(\lambda)^{c}}|n(y, x)-n(x, y)| m(d y)=:(\mathrm{I})+(\mathrm{II})+(\mathrm{III}) .
\end{aligned}
$$

It follows from $(2.4),(2.5)$ and (2.6) that

$$
(\mathrm{I})+(\mathrm{III}) \leq \frac{\varepsilon}{3 M} \sup _{x \in X} \int_{y \neq x}|n(y, x)-n(x, y)| m(d y)=\frac{\varepsilon}{3}
$$

and

$$
\begin{aligned}
(\mathrm{II})= & \int_{B_{x}(\lambda)^{c} \cap K}|u(y) \| n(y, x)-n(x, y)| m(d y) \\
& +\int_{B_{x}(\lambda)^{c} \cap K^{c}}|u(y)||n(y, x)-n(x, y)| m(d y) \\
\leq & m(K)\|u\|_{\infty} \sup _{x \in L^{c}} \sup _{y \in K}(n(x, y)+n(y, x))+M \sup _{y \in K^{c}}|u(y)|<\frac{2}{3} \varepsilon,
\end{aligned}
$$

which implies the desired result.

We should note that $\tilde{\mathcal{L}}$ defined in $(2.2)$ is not the 'adjoint operator' of $\mathcal{L}$ on $L^{2}(X ; m)$. In fact, if we denote by $\mathcal{L}^{*}$ the (formal) adjoint operator of $\mathcal{L}$ on $L^{2}(X ; m)$, then we have the following relation:

$$
(\mathcal{L} u, v)_{L^{2}(X ; m)}=(u, \tilde{\mathcal{L}} v)_{L^{2}(X ; m)}+(u, K v)_{L^{2}(X ; m)}, \quad u, v \in \mathcal{C} \cap L^{2}(X ; m),
$$

that is, $\mathcal{L}^{*} u(x)=\tilde{\mathcal{L}} u(x)+u(x) \cdot K(x)$. Here $(u, v)_{L^{2}(X ; m)}=\int_{X} u(x) v(x) m(d x)$ and

$$
K(x)=\int_{y \neq x}(n(y, x)-n(x, y)) m(d y), \quad x \in X .
$$

Since the function $K$ may take both positive and negative values, we do not know whether $\mathcal{L}^{*}$ satisfies the positive maximum principle. Thus $\mathcal{L}^{*}$ may not be the generator of a sub-Markov semigroup.

\section{Applications}

There are many jump-type Markov processes on $\mathbb{R}^{d}$ for which the corresponding generators are the following form:

$$
\mathcal{L} u(x)=\int_{y \neq x}\left(u(y)-u(x)-\nabla u(x) \cdot(y-x) \mathbf{1}_{B(1)}(y-x)\right) n(x, y) d y
$$


where $n(x, y)$ is a positive measurable function defined on the off-diagonal set, $B(r)$ is the closed ball at the origin with radius $r$ and $\mathbf{1}_{B(r)}$ is the indicator function of $B(r)$.

Conversely, if we are given an operator of the type (3.1), then it is natural to ask whether the operator generates a nice Markov process. Related to this problem, many people have considered the martingale problem for operators of this type (see the paper [3] and the books [16] for the references and related topics). However, so far, no one has obtained general conditions on $n$ that guarantee the existence or the uniqueness for the problem. In the sequel, instead of the operator (3.1), we consider the following integro-differential operator:

$$
\mathcal{L} u(x)=\int_{y \neq x}(u(y)-u(x)) n(x, y) d y \quad \text { for } u \in C_{0}^{1}\left(\mathbb{R}^{d}\right),
$$

where $C_{0}^{1}\left(\mathbb{R}^{d}\right)$ stands for the set of all continuously differentiable functions on $\mathbb{R}^{d}$ with compact support. As in $\S 2$, let $n$ be a positive measurable function defined on the off-diagonal set such that $\mathcal{L} u \in C_{\infty}\left(\mathbb{R}^{d}\right)$ for any $u \in C_{0}^{1}\left(\mathbb{R}^{d}\right)$. We further assume that $\mathcal{L}$ is closable on $C_{\infty}\left(\mathbb{R}^{d}\right)$ and its closure is the infinitesimal generator of a Feller semigroup on $C_{\infty}\left(\mathbb{R}^{d}\right)$. As stated above, if $k(x, d y)=n(x, y) d y$ is a bounded continuous kernel, then $\mathcal{L}$ is a bounded linear operator on $C_{\infty}\left(\mathbb{R}^{d}\right)$ satisfying the Feller property. On the other hand, if we take $n(x, y)=c(d, \alpha)|x-y|^{-d-\alpha}$ with $0<\alpha<1$, then $\mathcal{L}$ is nothing but $-(-\Delta)^{\alpha / 2}$ and this generates a symmetric $\alpha$-stable process on $\mathbb{R}^{d}$, where $c(d, \alpha)$ is an appropriate constant (see e.g. [18, p.217, Example 32.7]).

Corresponding to the operator $\mathcal{L}$, we consider the following carré du champ operator $\Gamma(u, v)$ for $u, v \in C_{0}^{1}\left(\mathbb{R}^{d}\right)$ as in $[23]$ :

$$
\Gamma(u, v)(x)=\mathcal{L}(u \cdot v)(x)-\mathcal{L} u(x) \cdot v(x)-u(x) \cdot \mathcal{L} v(x), \quad x \in \mathbb{R}^{d} .
$$

Then it follows from [23, Theorem 1] that

$$
\Gamma(u, v)(x)=\int_{y \neq x}(u(x)-u(y))(v(x)-v(y)) n(x, y) d y .
$$

Moreover, we can associate the symmetric quadratic form on $L^{2}\left(\mathbb{R}^{d}\right)$ :

$$
\begin{aligned}
\mathcal{E}(u, v) & =\int_{\mathbb{R}^{d}} \Gamma(u, v)(x) d x \\
& =\iint_{\mathbb{R}^{d} \times \mathbb{R}^{d} \backslash D}(u(x)-u(y))(v(x)-v(y)) n(x, y) d x d y,
\end{aligned}
$$

where $D=\left\{(x, x) \in \mathbb{R}^{d} \times \mathbb{R}^{d}\right\}$. Here it should be emphasized that we can not apply the theory of carré du champ operators developed by [7] and [12] to the operator $\mathcal{L}$ because the kernel $n(x, y) d y$ is not necessarily symmetric. In addition, our presentation above gives us a direct relation between the generator and the Dirichlet form. 
We now consider whether we can find a Feller process associated to $\mathcal{E}$ from the equivalence class by using the Feller property of $\mathcal{L}$. As for the regularity of the form, we have the following: $\mathcal{D}(\mathcal{E})=\left\{u \in L^{2}\left(\mathbb{R}^{d}\right): \mathcal{E}(u, u)<\infty\right\}$ contains $C_{0}^{1}\left(\mathbb{R}^{d}\right)$ if and only if

$$
\int_{y \neq x}(1 \wedge|x-y|) \nu(x, y) d y \in L_{\mathrm{loc}}^{1}\left(\mathbb{R}^{d}\right),
$$

where $\nu(x, y)=n(x, y)+n(y, x)$ (see [12, Example 1.2.4.], [22]). So, under this condition, $\left(\mathcal{E}, C_{0}^{1}\left(\mathbb{R}^{d}\right)\right)$ is a closable Markovian symmetric form on $L^{2}\left(\mathbb{R}^{d}\right)$ and there exists a symmetric Hunt process associated with the closure of the form, which is denoted by $(\mathcal{E}, \mathcal{F})$. Next we make a stronger condition on $n$ in order to consider a relation between the generator $\mathcal{L}$ and the Dirichlet form $(\mathcal{E}, \mathcal{F})$. To this end, we denote by $(\mathcal{A}, \mathcal{D}(\mathcal{A}))$ the $L^{2}$-generator of $(\mathcal{E}, \mathcal{F})$. We assume that

$\int_{y \neq x}(1 \wedge|x-y|) \nu(x, y) d y \in L_{\mathrm{loc}}^{2}\left(\mathbb{R}^{d}\right)$ and $\int_{B(r)} \nu(x, y) d y \in L^{2}\left(\mathbb{R}^{d} \backslash B(R)\right)$ for any $r, R>0$ with $R-r>1$. It is then showed in [22, Theorem 4.1] that $C_{0}^{1}\left(\mathbb{R}^{d}\right) \subset \mathcal{D}(\mathcal{A})$ and

$$
\mathcal{A} u(x)=\int_{y \neq x}(u(y)-u(x)) \nu(x, y) d y \quad \text { for } u \in C_{0}^{1}\left(\mathbb{R}^{d}\right) .
$$

Let

$$
\tilde{\mathcal{L}} u(x)=\int_{y \neq x}(u(y)-u(x)) n(y, x) d y \quad \text { for } u \in C_{0}^{1}\left(\mathbb{R}^{d}\right)
$$

and

$$
B u(x)=\int_{y \neq x}(u(y)-u(x))(n(y, x)-n(x, y)) d y .
$$

We can then restate Theorem 2.2 for this setting as follows:

Corollary 3.1. Assume that $\mathcal{L}$ given by $(3.2)$ is closable on $C_{\infty}\left(\mathbb{R}^{d}\right)$ and its closure is the infinitesimal generator of a Feller semigroup on $C_{\infty}\left(\mathbb{R}^{d}\right)$, and that (3.3) holds. Assume further that $B$ is a bounded linear operator on $C_{\infty}\left(\mathbb{R}^{d}\right)$. Then $\left.\mathcal{A}\right|_{C_{0}^{1}\left(\mathbb{R}^{d}\right)}$ and $\tilde{\mathcal{L}}$ are also closable on $C_{\infty}\left(\mathbb{R}^{d}\right)$ and their closures are the infinitesimal generators of Feller semigroups on $C_{\infty}\left(\mathbb{R}^{d}\right)$, respectively.

REMARK 3.2. (i) Fukushima and Stroock ([13]) discussed a relationship between a Markov process as a solution of the martingale problem and one associated with a (symmetric) Dirichlet form. In particular, their results are applicable to our setting: the closable operator $\left.\mathcal{A}\right|_{C_{0}^{1}\left(\mathbb{R}^{d}\right)}$ on $C_{\infty}\left(\mathbb{R}^{d}\right)$ and the Dirichlet form $(\mathcal{E}, \mathcal{F})$. So it follows from [13, Theorem 2.9] that a symmetric Hunt process associated with $(\mathcal{E}, \mathcal{F})$ has a version that is Feller if the conditions in Corollary 3.1 are satisfied. 
(ii) When the kernel $k(x, d y)=n(x, y) d y$ is not bounded, we do not know general conditions on $n$ so that the closure of $\mathcal{L}$ generates a nice Markov process as mentioned before. However, if we consider the quadratic form defined by

$$
\eta(u, v)=-(u, \mathcal{L} v)_{L^{2}\left(\mathbb{R}^{d}\right)}=-\iint_{\mathbb{R}^{d} \times \mathbb{R}^{d} \backslash D} u(x)(v(y)-v(x)) n(x, y) d y d x
$$

for some nice functions $u, v$, then we will be able to show that this quadratic form becomes a (non-symmetric) regular Dirichlet form under an appropriate condition on $n$ (see [24]).

EXAMPLE 3.3. (Cauchy distribution). Let $\varphi(x)$ be a strictly positive continuous function on $|x| \leq 1$ and $\psi(x)$ a strictly positive continuous function on $|x| \geq 1$ satisfying

$$
\int_{|x|>1} \frac{1}{\psi(x)^{d / 2}} d x<\infty .
$$

Let $c_{d}=\Gamma((d+1) / 2) / \pi^{(d+1) / 2}$ and

$$
n(x, y)= \begin{cases}c_{d} \cdot \frac{\varphi(x)^{d / 2}}{\left(\varphi(x)|x-y|^{2}+1\right)^{(d+1) / 2}}, & |x| \leq 1 \\ c_{d} \cdot \frac{\sqrt{\psi(x)}}{\left(|x-y|^{2}+\psi(x)\right)^{(d+1) / 2}}, & |x|>1 .\end{cases}
$$

Here note that for $t>0$,

$$
p_{t}(x)=c_{d} \frac{t}{\left(|x|^{2}+t^{2}\right)^{(d+1) / 2}}
$$

is the transition function of a $d$-dimensional Cauchy distribution. Then

$$
\mathcal{L} u(x)=\int_{y \neq x}(u(y)-u(x)) n(x, y) d y
$$

defines a bounded linear operator on $C_{\infty}\left(\mathbb{R}^{d}\right)$ and generates a Feller semigroup on $C_{\infty}\left(\mathbb{R}^{d}\right)$. We assume that $n$ is continuous on $\mathbb{R}^{d} \times \mathbb{R}^{d} \backslash D$.

We now prove that $n$ satisfies the conditions in Corollary 3.1. We first show that (3.3) is satisfied. For a compact set $K \subset \mathbb{R}^{d}$,

$$
\begin{aligned}
& \int_{K}\left(\int_{y \neq x}(1 \wedge|x-y|)(n(x, y)+n(y, x)) d y\right)^{2} d x \\
& \leq 2 \int_{K}\left(\int_{y \neq x}(1 \wedge|x-y|) n(x, y) d y\right)^{2} d x \\
& \quad+2 \int_{K}\left(\int_{y \neq x}(1 \wedge|x-y|) n(y, x) d y\right)^{2} d x=: 2((\mathrm{I})+(\mathrm{II})) .
\end{aligned}
$$


Since $\int_{\mathbb{R}^{d}} n(x, y) d y=1$ for any $x \neq 0$, we have $(\mathrm{I})<\infty$. For (II), if we denote by $|\cdot|$ the $d$-dimensional Lebesgue measure, then the estimate

$$
\begin{aligned}
(\mathrm{II}) \leq & 2 c_{d}^{2} \int_{K}\left(\int_{|y| \leq 1} \frac{\varphi(y)^{d / 2}}{\left(\varphi(y)|x-y|^{2}+1\right)^{(d+1) / 2}} d y\right)^{2} d x \\
& +2 c_{d}^{2} \int_{K}\left(\int_{|y|>1} \frac{\sqrt{\psi(y)}}{\left(|x-y|^{2}+\psi(y)\right)^{(d+1) / 2}} d y\right)^{2} d x \\
\leq & 2 c_{d}^{2}|K|\left\{\left(\int_{|y| \leq 1} \varphi(y)^{d / 2} d y\right)^{2}+\left(\int_{|y|>1} \frac{1}{\psi(y)^{d / 2}} d y\right)^{2}\right\}<\infty
\end{aligned}
$$

holds, whence the first condition in (3.3) is satisfied. Take $r, R>0$ so that $R-r>1$. Then since $|x-y|^{d} \nu(x, y)$ is bounded, we have

$$
\begin{aligned}
\int_{|x|>R}\left(\int_{|y| \leq r} \nu(x, y) d y\right)^{2} d x \\
\quad=\int_{|x|>R}\left(\int_{|y| \leq r}|x-y|^{d} \nu(x, y) \frac{1}{|x-y|^{d}} d y\right)^{2} d x \\
\leq M_{d, r} \int_{|x|>R} \frac{1}{(|x|-r)^{2 d}} d x<\infty,
\end{aligned}
$$

where $M_{d, r}$ is a positive constant depending only on $d$ and $r$. This shows that the second condition in (3.3) is satisfied.

We next show that the operator $B$ defined by

$$
B u(x)=\int_{y \neq x}(u(y)-u(x))(n(y, x)-n(x, y)) d y
$$

is a bounded linear operator on $C_{\infty}\left(\mathbb{R}^{d}\right)$ by checking Conditions (1)-(4) in Remark 2.3. Condition (1) is obviously satisfied. We now check Condition (2). If we fix $\delta \geq 1$, then

$$
\int_{y \neq x}|n(y, x)-n(x, y)|^{\delta} d y \leq 2^{\delta-1} \int_{y \neq x}\left(n(x, y)^{\delta}+n(y, x)^{\delta}\right) d y .
$$

If $|x| \leq 1$, then it follows by a change of variables that

$$
\begin{aligned}
\int_{y \neq x} n(x, y)^{\delta} d y & =c_{d}^{\delta} \int_{y \neq x} \frac{\varphi(x)^{d \delta / 2}}{\left(\varphi(x)|x-y|^{2}+1\right)^{(d+1) \delta / 2}} d y \\
& =c_{d}^{\delta} \varphi(x)^{d(\delta-1) / 2} \int_{0}^{\infty} \frac{r^{d-1}}{\left(1+r^{2}\right)^{(d+1) \delta / 2}} d r \\
& \leq c_{d}^{\delta}\left(\sup _{|x| \leq 1} \varphi(x)^{d(\delta-1) / 2}\right) \int_{0}^{\infty} \frac{r^{d-1}}{\left(1+r^{2}\right)^{(d+1) \delta / 2}} d r<\infty
\end{aligned}
$$


By the same way, we find that if $|x|>1$, then

$$
\begin{aligned}
\int_{y \neq x} n(x, y)^{\delta} d y & =c_{d}^{\delta} \frac{1}{\psi(x)^{d(\delta-1) / 2}} \int_{0}^{\infty} \frac{r^{d-1}}{\left(1+r^{2}\right)^{(d+1) \delta / 2}} d r \\
& \leq c_{d}^{\delta}\left(\inf _{|x|>1} \psi(x)^{d(\delta-1) / 2}\right)^{-1} \int_{0}^{\infty} \frac{r^{d-1}}{\left(1+r^{2}\right)^{(d+1) \delta / 2}} d r<\infty .
\end{aligned}
$$

We also see that

$$
\begin{aligned}
\int_{y \neq x} n(y, x)^{\delta} d y= & c_{d}^{\delta} \int_{|y| \leq 1} \frac{\varphi(y)^{d \delta / 2}}{\left(\varphi(y)|y-x|^{2}+1\right)^{(d+1) \delta / 2}} d y \\
& +c_{d}^{\delta} \int_{|y|>1} \frac{\psi(y)^{\delta / 2}}{\left(|y-x|^{2}+\psi(y)\right)^{(d+1) \delta / 2}} d y \\
\leq & c_{d}^{\delta}\left(\int_{|y| \leq 1} \varphi(y)^{d \delta / 2} d y+\int_{|y|>1} \frac{1}{\psi(y)^{d \delta / 2}} d y\right)<\infty
\end{aligned}
$$

whence Condition (2) holds.

We next check Condition (3). For $r>0$, let us denote by $B_{x}(r)$ a closed ball with radius $r$ centered at $x \in \mathbb{R}^{d}$ and $B(r):=B_{0}(r)$. Then for any compact set $K \subset \mathbb{R}^{d}$, there exists $N \in \mathbb{N}$ such that $K \subset B(N)$. Take $F_{m}=B(m)$ and let $n \geq N+1$. We then see that $|y-x| \geq|y|-|x| \geq n-N$ for any $x \in K$ and $y \in F_{n}^{c}$. Hence if $x \in K \cap B(1)^{c}$, then

$$
\begin{aligned}
\int_{F_{n}^{c}} n(x, y) d y & \leq c_{d} \int_{B_{x}(n-N)^{c}} \frac{\sqrt{\psi(x)}}{\left(|x-y|^{2}+\psi(x)\right)^{(d+1) / 2}} d y \\
& =c_{d} \int_{(n-N) / \psi(x)}^{\infty} \frac{r^{d-1}}{\left(1+r^{2}\right)^{(d+1) / 2}} d r \\
& \leq c_{d} \int_{(n-N) / \inf _{|x|>1} \psi(x)}^{\infty} \frac{r^{d-1}}{\left(1+r^{2}\right)^{(d+1) / 2}} d r \longrightarrow 0 \text { as } n \rightarrow \infty
\end{aligned}
$$

that is,

$$
\lim _{n \rightarrow \infty} \sup _{x \in K \cap B(1)^{c}} \int_{F_{n}^{c}} n(x, y) d y=0 .
$$

In a similar way to this, we see that

$$
\lim _{n \rightarrow \infty} \sup _{x \in K \cap B(1)} \int_{F_{n}^{c}} n(x, y) d y=0 .
$$

Noting that $B(1) \subset F_{n}$, we get

$$
\begin{aligned}
\sup _{x \in K} \int_{F_{n}^{c}} n(y, x) d y & =c_{d} \sup _{x \in K} \int_{F_{n}^{c}} \frac{\sqrt{\psi(y)}}{\left(|y-x|^{2}+\psi(y)\right)^{(d+1) / 2}} d y \\
& \leq c_{d} \int_{F_{n}^{c}} \frac{1}{\psi(y)^{d / 2}} d y \longrightarrow 0 \text { as } n \rightarrow \infty .
\end{aligned}
$$


Combining the arguments above with the inequality

$$
\int_{F_{n}^{c}}|n(x, y)-n(y, x)| d y \leq \int_{F_{n}^{c}}(n(x, y)+n(y, x)) d y,
$$

we find Condition (3) holds.

We finally check Condition (4). Let $K$ and $N$ be the same as before. For any $\varepsilon>0$, we take $n>\left(2 c_{d} / \varepsilon\right)^{1 / d}+N$. Then a direct calculation implies that

$$
n(x, y) \leq \frac{c_{d}}{|x-y|^{d}} \leq \frac{c_{d}}{(n-N)^{d}}<\frac{\varepsilon}{2} \quad \text { for any } x \in K \text { and } y \in B(n)^{c} .
$$

In addition, we see that $n(y, x)$ is also less than $\varepsilon / 2$ for any $x \in K$ and $y \in B(n)^{c}$, which concludes that Condition (4) is satisfied by taking $L=B(n)$.

As a result of the arguments above, the operators

$$
\mathcal{A} u(x)=\int_{y \neq x}(u(y)-u(x))(n(x, y)+n(y, x)) d y \quad \text { for } u \in C_{0}^{1}\left(\mathbb{R}^{d}\right)
$$

and

$$
\tilde{\mathcal{L}} u(x)=\int_{y \neq x}(u(y)-u(x)) n(y, x) d y \quad \text { for } u \in C_{0}^{1}\left(\mathbb{R}^{d}\right)
$$

are closable on $C_{\infty}\left(\mathbb{R}^{d}\right)$ and their closures generate Feller semigroups on $C_{\infty}\left(\mathbb{R}^{d}\right)$, respectively. Moreover, put

$$
\begin{aligned}
k(x, y):= & \left(\frac{\varphi(x)^{d / 2}}{\left(\varphi(x)|x-y|^{2}+1\right)^{(d+1) / 2}} \mathbf{1}_{\{|x| \leq 1\}}\right. \\
& \left.+\frac{\sqrt{\psi(x)}}{\left(|x-y|^{2}+\psi(x)\right)^{(d+1) / 2}} \mathbf{1}_{\{|x|>1\}}\right),
\end{aligned}
$$

and define

$$
\mathcal{E}(u, v)=c_{d} \iint_{\mathbb{R}^{d} \times \mathbb{R}^{d} \backslash D}(u(x)-u(y))(v(x)-v(y)) k(x, y) d x d y .
$$

Then, setting $\mathcal{F}=\overline{C_{0}^{1}\left(\mathbb{R}^{d}\right)} \sqrt{\mathcal{E}_{1}(\cdot, \cdot)}$, we see that $(\mathcal{E}, \mathcal{F})$ is a regular Dirichlet form and the corresponding symmetric Hunt process can be refined to be a Feller process.

Before introducing another example, we give a simple but useful remark. Let $w$ be a bounded continuous function defined on $\mathbb{R}^{d}$ so that for some $\lambda>0$,

$$
\lambda \leq w(x) \leq \lambda^{-1} \quad \text { for all } x \in \mathbb{R}^{d},
$$

and define

$$
\mathcal{L}_{w} u(x):=w(x) \mathcal{L} u(x)=w(x) \int_{y \neq x}(u(y)-u(x)) n(x, y) d y \quad \text { for } u \in C_{0}^{1}\left(\mathbb{R}^{d}\right),
$$

where $\mathcal{L}$ is the operator given by (3.2). If all the conditions in Corollary 3.1 are satisfied, then $\mathcal{L}_{w}$ satisfies the positive maximum principle, and hence is 
dissipative. So, we can conclude that $\mathcal{L}_{w}$ is also the generator of a Feller semigroup. In particular, the Feller process associated with $\mathcal{L}_{w}$ is a time changed process of that associated with $\mathcal{L}$.

EXAMPLE 3.4. (stable-like process). Let $\alpha(x)$ be a positive measurable function on $\mathbb{R}^{d}$. Define for $u \in C_{0}^{2}\left(\mathbb{R}^{d}\right)$,

$$
\begin{aligned}
\mathcal{L}_{w} u(x) & :=w(\alpha(x)) \mathcal{L} u(x) \\
& :=w(\alpha(x)) \int_{h \neq 0} \frac{u(x+h)-u(x)-\nabla u(x) \cdot h \mathbf{1}_{\{|h| \leq 1\}}(h)}{|h|^{d+\alpha(x)}} d h,
\end{aligned}
$$

where

$$
w(\alpha)=\frac{\Gamma(1+\alpha / 2) \Gamma((\alpha+d) / 2) \sin ((\pi \alpha) / 2)}{2^{1-\alpha} \pi^{d / 2+1}} .
$$

Then $\mathcal{L}_{w} e^{i u \cdot x}=-|u|^{\alpha(x)} e^{i u \cdot x}$ holds (see e.g. [1, p.402]).

Assume that

$$
0<\inf _{x \in \mathbb{R}^{d}} \alpha(x) \leq \sup _{x \in \mathbb{R}^{d}} \alpha(x)<2,
$$

and that for some $M>0$,

$$
|\alpha(x)-\alpha(y)| \leq M|x-y| \text { for } x, y \in \mathbb{R}^{d} .
$$

Bass ([2]) then showed that there exists a unique strong Markov and Feller process $\mathbf{M}=\left(X_{t}, P_{x}\right)$ such that $P_{x}$ solves the martingale problem for $\mathcal{L}_{w}$ at every starting point $x \in \mathbb{R}^{d}$. Here we can see from (3.5) that if $\alpha$ satisfies the conditions (3.6) and $(3.7)$, then $w(\alpha(\cdot))$ is a bounded continuous function and is also bounded below by some positive constant. Hence $\mathcal{L}=(1 / w(\alpha(\cdot))) \mathcal{L}_{w}$ is also the generator of a Feller semigroup. So, in the sequel we consider the operator $\mathcal{L}$ for simplicity. We now keep (3.7) and assume (3.8) instead of $(3.6)$ :

$$
0<\inf _{x \in \mathbb{R}^{d}} \alpha(x) \leq \sup _{x \in \mathbb{R}^{d}} \alpha(x)<1 .
$$

Then for $u \in C_{0}^{1}\left(\mathbb{R}^{d}\right)$, we can reduce the form of $\mathcal{L} u$ as follows:

$$
\begin{aligned}
\mathcal{L} u(x) & =\int_{h \neq 0}(u(x+h)-u(x))|h|^{-d-\alpha(x)} d h \\
& =\int_{y \neq x}(u(y)-u(x))|x-y|^{-d-\alpha(x)} d y .
\end{aligned}
$$

Namely, this is the case $n(x, y)=|x-y|^{-d-\alpha(x)}$ in (3.2). Note that (3.8) implies (3.3) as mentioned in [22, Example 4.1].

We now show that the operator $B$ defined by

$$
B u(x)=\int_{h \neq 0}(u(x+h)-u(x))\left(|h|^{-d-\alpha(x+h)}-|h|^{-d-\alpha(x)}\right) d h
$$


is a bounded linear operator on $C_{\infty}\left(\mathbb{R}^{d}\right)$ directly. In fact, we first see that

$$
\begin{aligned}
\left.|| h\right|^{-\alpha(x)}-|h|^{-\alpha(x+h)} \mid & =\left.\left|\int_{\alpha(x+h)}^{\alpha(x)}\right| h\right|^{-u} \log \frac{1}{|h|} d u \mid \\
& \leq|\alpha(x+h)-\alpha(x)||h|^{-(\alpha(x) \vee \alpha(x+h))} \log \frac{1}{|h|} \\
& \leq M|h|^{1-\bar{\alpha}} \log \frac{1}{|h|} \quad \text { for } 0<|h|<1
\end{aligned}
$$

in a similar way to [19, Example 2.6] (see also the proof of [15, Lemma 3.1]) and that $\left.|| h\right|^{-\alpha(x)}-\left.|h|^{-\alpha(x+h)}|\leq 2| h\right|^{-\underline{\alpha}}$ for $|h|>1$, where

$$
0<\underline{\alpha}:=\inf _{x \in \mathbb{R}^{d}} \alpha(x) \leq \bar{\alpha}:=\sup _{x \in \mathbb{R}^{d}} \alpha(x)<1 .
$$

So the absolute value of the integrand in the right hand side of (3.9) is dominated by

$$
c\|u\|_{\infty}\left(\mathbf{1}_{\{|h| \leq 1\}} \cdot|h|^{1-d-\bar{\alpha}} \log (1 /|h|)+\mathbf{1}_{\{|h|>1\}} \cdot|h|^{-d-\underline{\alpha}}\right)
$$

for some constant $c>0$ independent to $x$ and $h$, which is integrable on $\{h \neq 0\}$ with respect to the Lebesgue measure. Consequently, by virtue of the dominated convergence theorem, we find that $B$ is a bounded linear operator on $C_{\infty}\left(\mathbb{R}^{d}\right)$.

Let

$\mathcal{E}(u, v)=\iint_{h \neq 0} \frac{(u(x+h)-u(x))(v(x+h)-v(x))}{|h|^{d+\alpha(x)}} d h d x \quad$ for $u, v \in C_{0}^{1}\left(\mathbb{R}^{d}\right)$

and $\mathcal{F}={\overline{C_{0}^{1}\left(\mathbb{R}^{d}\right)}}^{\sqrt{\mathcal{E}_{1}(\cdot, \cdot)}}$. Then $(\mathcal{E}, \mathcal{F})$ generates a symmetric stable-like process introduced in [21]. Moreover, if we denote by $\mathcal{A}$ the $L^{2}\left(\mathbb{R}^{d}\right)$-generator of $(\mathcal{E}, \mathcal{F})$, then Corollary 3.1 shows that

$$
\left.\mathcal{A}\right|_{C_{0}^{1}\left(\mathbb{R}^{d}\right)} u(x)=\int_{h \neq 0}(u(x+h)-u(x))\left(|h|^{-d-\alpha(x)}+|h|^{-d-\alpha(x+h)}\right) d h
$$

is closable on $C_{\infty}\left(\mathbb{R}^{d}\right)$ and its closure generates a Feller semigroup on $C_{\infty}\left(\mathbb{R}^{d}\right)$. Namely, the corresponding symmetric stable-like process can be refined to be a Feller process. We see that

$$
\tilde{\mathcal{L}} u(x)=\int_{h \neq 0}(u(x+h)-u(x))|h|^{-d-\alpha(x+h)} d h \quad \text { for } u \in C_{0}^{1}\left(\mathbb{R}^{d}\right)
$$

is also closable on $C_{\infty}\left(\mathbb{R}^{d}\right)$ and its closure generates a Feller process.

Finally, we consider the following operator:

$$
\mathcal{L}_{1} u(x)=\int_{h \neq 0}(u(x+h)-u(x)) \frac{c(x, h)}{|h|^{d+\alpha(x)}} d h \quad \text { for } u \in C_{0}^{1}\left(\mathbb{R}^{d}\right),
$$


where $c(x, h)$ is a bounded, nonnegative and continuous function defined on $\mathbb{R}^{d} \times \mathbb{R}^{d}$. We assume that there exist positive constants $M>0$ and $\delta>0$ with $\bar{\alpha}<\delta \leq 1$ such that

$$
|c(x, h)-1| \leq M|h|^{\delta} \quad \text { for } x \in \mathbb{R}^{d} \text { and }|h| \leq 1 .
$$

We can then write down $\mathcal{L}_{1}$ as follows:

$$
\begin{aligned}
\mathcal{L}_{1} u(x)= & \int_{h \neq 0}(u(x+h)-u(x)) \frac{1}{|h|^{d+\alpha(x)}} d h \\
& +\int_{h \neq 0}(u(x+h)-u(x)) \frac{c(x, h)-1}{|h|^{d+\alpha(x)}} d h \\
=: & \mathcal{L} u(x)+B_{1} u(x) \quad \text { for } x \in \mathbb{R}^{d} .
\end{aligned}
$$

Using the assumption for $c(x, h)$, we can easily show that $B_{1}$ is a bounded linear operator on $C_{\infty}\left(\mathbb{R}^{d}\right)$. Therefore, we see from Proposition 2.1 that $\mathcal{L}_{1}$ is closable and its closure generates a Feller semigroup on $C_{\infty}\left(\mathbb{R}^{d}\right)(c . f$. Theorem 5.1 and 5.2 in [4]).

Let

$$
\begin{array}{r}
\mathcal{G}(u, v)=\iint_{h \neq 0}(u(x+h)-u(x))(v(x+h)-v(x)) \frac{c(x, h)}{\mid h^{d+\alpha(x)}} d h d x \\
\text { for } u, v \in C_{0}^{1}\left(\mathbb{R}^{d}\right)
\end{array}
$$

and $\mathcal{H}={\overline{C_{0}^{1}\left(\mathbb{R}^{d}\right)}}^{\sqrt{\mathcal{G}_{1}(\cdot, \cdot)}}$. Then $(\mathcal{G}, \mathcal{H})$ is a regular Dirichlet form and generates a jump-type symmetric Markov process. Let $\left(\mathcal{A}_{1}, \mathcal{D}\left(\mathcal{A}_{1}\right)\right)$ be the $L^{2}\left(\mathbb{R}^{d}\right)$ generator of $(\mathcal{G}, \mathcal{H})$. Then

$$
\left.\mathcal{A}_{1}\right|_{C_{0}^{1}\left(\mathbb{R}^{d}\right)} u(x)=\int_{h \neq 0}(u(x+h)-u(x))\left(\frac{c(x, h)}{|h|^{d+\alpha(x)}}+\frac{c(x+h,-h)}{|h|^{d+\alpha(x+h)}}\right) d h .
$$

Let us define the operator $\tilde{B}_{1}$ by

$$
\tilde{B}_{1} u(x)=\int_{h \neq 0}(u(x+h)-u(x)) \frac{c(x+h,-h)-1}{|h|^{d+\alpha(x+h)}} d h .
$$

Then $\tilde{B}_{1}$ is also a bounded linear operator on $C_{\infty}\left(\mathbb{R}^{d}\right)$. Since

$$
\left.\mathcal{A}_{1}\right|_{C_{0}^{1}\left(\mathbb{R}^{d}\right)} u(x)=\left.\mathcal{A}\right|_{C_{0}^{1}\left(\mathbb{R}^{d}\right)} u(x)+B_{1} u(x)+\tilde{B}_{1} u(x) \text { for } x \in \mathbb{R}^{d},
$$

Proposition 2.1 implies that $\left.\mathcal{A}_{1}\right|_{C_{0}^{1}\left(\mathbb{R}^{d}\right)}$ is closable on $C_{\infty}\left(\mathbb{R}^{d}\right)$ and its closure generates a Feller semigroup on $C_{\infty}\left(\mathbb{R}^{d}\right)$. Hence a Markov process corresponding to $(\mathcal{G}, \mathcal{H})$ can be refined to be a Feller process. Set

$$
\tilde{\mathcal{L}}_{1} u(x)=\int_{h \neq 0}(u(x+h)-u(x)) \frac{c(x+h,-h)}{|h|^{d+\alpha(x+h)}} d h \quad \text { for } u \in C_{0}^{1}\left(\mathbb{R}^{d}\right) .
$$

By noting that

$$
\tilde{\mathcal{L}}_{1} u(x)=\tilde{\mathcal{L}} u(x)+\tilde{B}_{1} u(x) \quad \text { for } x \in \mathbb{R}^{d}
$$


and for $u \in C_{0}^{1}\left(\mathbb{R}^{d}\right)$, the operator $\tilde{\mathcal{L}}_{1}$ is also closable on $C_{\infty}\left(\mathbb{R}^{d}\right)$ and its closure generates a Feller semigroup on $C_{\infty}\left(\mathbb{R}^{d}\right)$.

REMARK 3.5. Because of the restricted conditions on $n$, we can only show the Feller property of the processes corresponding to the form (3.10) under (3.7) and (3.8). So, the general case (3.6) still remains open.

\section{ACKNOWLEDGEMENTS.}

The authors would like to thank Professor Masatoshi Fukushima for letting them know the paper [13]. They would be grateful to two referees for valuable comments and suggestions, which improved this paper.

\section{REFERENCES}

[1] N. Aronszajn and K. T. Smith, Theory of Bessel potentials. I, Ann. Inst. Fourier (Grenoble) 11 (1961), 385-475.

[2] R. F. Bass, Uniqueness in law for pure jump Markov processes, Probab. Theory Related Fields 79 (1988), 271-287.

[3] R. F. Bass, Stochastic differential equations with jumps, Probab. Surv. 1 (2004), $1-19$.

[4] R. F. Bass, SDEs with jumps, Lecture notes for Cornell Summer School in Proba-

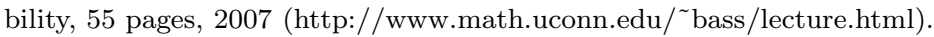

[5] R. F. Bass and M. Kassmann, Hölder continuity of harmonic functions with respect to operators of variable order, Comm. Partial Differential Equations 30 (2005), 12491259.

[6] R. F. Bass and D. A. Levin, Harnack inequalities for jump processes, Potential Anal. 17 (2002), 375-388.

[7] N. Bouleau and F. Hirsch, Dirichlet forms and analysis on Wiener space, Walter de Gruyter, Berlin, 1991.

[8] Z.-Q. Chen and T. Kumagai, Heat kernel estimates for stable-like processes on d-sets, Stochastic Process. Appl. 108 (2003), 27-62.

[9] E. B. Davies, One-parameter semigroups, Academic Press, London, 1980.

[10] S. N. Ethier and T. G. Kurtz, Markov Processes. Characterization and Convergence, Wiley, New York, 1986.

[11] M. Fukushima, Dirichlet spaces and strong Markov processes, Trans. Amer. Math. Soc. 162 (1971), 185-224.

[12] M. Fukushima, Y. Oshima and M. Takeda, Dirichlet forms and symmetric Markov processes, Walter de Gruyter, Berlin, 1994.

[13] M. Fukushima and D. Stroock, Reversibility of solutions to martingale problems, Probability, statistical mechanics, and number theory, Adv. Math. Suppl. Stud., 9, Academic Press, Orlando, 1986, 107-123.

[14] R. Husseini and M. Kassmann, Jump processes, $\mathcal{L}$-harmonic functions, continuity estimates and the Feller property, Ann. Inst. Henri Poincaré Probab. Stat. 45 (2009), 1099-1115.

[15] Y. Isozaki and T. Uemura, A family of symmetric stable-like processes and its global path properties, Probab. Math. Statist. 24 (2004), 145-164.

[16] N. Jacob, Pseudo Differential Operators and Markov Processes, Vol. 1-3, Imperial College Press, 2001-5.

[17] A. Pazy, Semigroups of linear operators and applications to partial differential equations, Springer-Verlag, New York, 1983. 
[18] K. Sato, Lévy processes and infinitely divisible distributions, Cambridge University Press, Cambridge, 1999.

[19] R. L. Schilling and T. Uemura, On the Feller property of Dirichlet forms generated by pseudo differential operators, Tohoku Math. J. (2) 59 (2007), 401-422.

[20] R. Song and Z. Vondraček, Harnack inequality for some classes of Markov processes, Math. Z. 246 (2004), 177-202.

[21] T. Uemura, On some path properties of symmetric stable-like processes for one dimension, Potential Anal. 16 (2002), 79-91.

[22] T. Uemura, On symmetric stable-like processes: some path properties and generators, J. Theoret. Probab. 17 (2004), 541-555.

[23] T. Uemura, A remark on non-local operators with variable order, Osaka J. Math. 46 (2009), 503-514.

[24] M. Fukushima and T. Uemura, Jump-type Hunt processes generated by lower bounded semi-Dirichlet forms, preprint, 2010.

Y. Shiozawa

College of Science and Engineering

Ritsumeikan University

Kusatsu Shiga 525-8577

Japan

Current Address:

Graduate School of Natural Science and Engineering

Okayama University

Okayama 700-8530

Japan

E-mail: shiozawa@ems.okayama-u.ac.jp

Toshihiro Uemura

School of Business Administration

University of Hyogo

Kobe 651-2197

Japan

Current Address:

Department of Mathematics

Faculty of Engineering Science

Kansai University

Suita, Osaka 564-8680

Japan

E-mail: uemura@iz.u-hyogo.ac.jp

E-mail: t-uemura@kansai-u.ac.jp

Received: 20.8.2008.

Revised: 8.1.2009. \& 17.3.2009. 